\title{
EFEITO DE GENÓTIPOS DE BATATA SOBRE Diabrotica speciosa (COLEOPTERA: CH RYSOMELIDAE) EM CONDIÇÕES DE CAMPO
}

\author{
Potato genotypes effect on Diabrotica speciosa \\ (C oleoptera: Chrysomelidae) in field onditions
}

\begin{abstract}
Rui Scaramella Furiatti ${ }^{\mathrm{a}}$
a Engenheiro Agrônomo, Professor adjunto do D epartamento deAgronomia, UniversidadeE stadual de Ponta G rossa (UEPG ), Ponta G rossa, PR - Brasil, e-mail: furiatti@ convoy.com.br
\end{abstract}

\section{Resumo}

Avaliou-se um clone de batata procedente da EMBRA PA-CNPH, em relação à resistência a D . speciosa, comparado a cultivares de mercado. Foram utilizado s cinco cultivares (Bintje, Jatte-Bintje, Monalisa, Atlantic e Crebella) e um clone (L 235-4). 0 delineamento experimental foi em blocos casualizados arranjados em parcelas subdivididas com 12 tratamentos (parcela principal dois tratamentos: com e sem controle de insetos com rotação de inseticidas e subparcelas com seis tratamentos: cinco cultivares mais um clone) e quatro repetições. Para a avaliação dos danos das larvas de D. speciosa foram contados os furos em 25 tubérculos coletados por parcela. Pôde-se concluir que: a cultivar Crebella apresentou 0 maior índice de desfolha e a cultivar Bintje o menor. Com relação aos danos ocasionados pelos adultos, 0 uso de inseticidas só é justificado na fase inicial da cultura, sendo a variedade Crebella a mais suscetível, e o clone (L235-4) o menos atacado; com relação aos danos nos tubérculos, a variedade Bintje foi a menos atacada e a Monalisa a mais atacada; o uso de inseticidas reduziu significativamente os danos nos tubérculos, eliminando as diferenças entre as cultivares e o clone de batata; não há relação entre os danos nas folhagens e os danos nos tubérculos.

Palavras-chave: Insecta. Solanum berthaultii. Resistência varietal.

\section{Abstract}

A potato clone from EMBRAPA-CNPH was evaluated in relation to its resistance against the D. speciosa compared to cultivate from the market. It was used five cultivates (Bintje, JatteBintje, Monalisa, Atlantic and Crebella) and a clone (L 235-4). The experiment organized as a splitplot in random blocks design with twelve treatments (the main part 2 treatments: with and 
without insect control with insecticide rotation and sub-parts with 6 treatments: five cultivates plus a clone) and four replicates for each treatment. In order to evaluate damages caused by the larva D. speciosa it was counted the holes in 25 tubercles colleted per part. We can conclude the Crebella cultivate was the most impacted, losing more leaves and the Bintje cultivate was the least impacted. In relation to occasional damages caused by adults the insecticide usage is only justified at the beginning of the culture being the cultivar Crebella the most attacked and the clone the least; in relation to damages on tubercles the Bintje cultivar was the least attacked and Monalisa the most attacked; the insecticides usage was only efficient on earlier phases of the culture for all cultivates including the clone, except for the Bintje cultivated and besides reduced significantly the tubercles damages, eliminating the differences between cultivates and potato clones; there is no relation between leaf damages and tubercles damages.

Keywords: Insecta. Solanum berthaultii. Varietal resistance.

\section{INTRODUÇÃO}

A batata apresenta uma série de problemas fitossanitários. Os insetos são os responsáveis por grandes danos que se iniciam no plantio e vão até o armazenamento. A vaquinha [D iabrotica speciosa (G erm. 1824) (Coleoptera: Chrysomelidae)], é uma das principais pragas desta cultura e ocasionam danos às folhagens e aos tubérculos.

$O$ adulto de $D$. speciosa é perigoso na fase inicial da cultura da batata, pois danifica folhas e hastes de plantas, causando redução da área foliar e favorecendo o aparecimento de doenças. As larvas danificam os tubérculos, desde o início da tuberização, podendo deixá-los completamente furados (perfurações de mais de $1 \mathrm{~mm}$ ). O s tubérculos danificados são chamados "alfinetados", não tem boa aparência e, por isso, apresentam menor valor comercial (HAJI, 1981).

Uma das alternativas para reduzir os danos dos insetos nas lavouras comerciais é o uso de variedades resistentes. A espécie de batata Solanum berthaultii (OCHOA; SCHMIEDICHE, 1983) foi reportada como resistente a pulgões. Em trabalhos subsequentes, Gibson e Turner (1977), Tingey e G ibson (1978) e Tingey e Sinden (1982) expandiram a lista de pragas para as quais S. berthaultii é resistente.

Lara, Poletti e Barbosa (2000a) avaliaram a resistência de genótipos de batata (Solanum spp.) a D . speciosa em condições de campo e laboratório. 0 genótipo 288.776-3 destacou-se como resistente às larvas e adultos de $\mathrm{D}$. speciosa, sendo a resistência de adultos do tipo não-preferência para alimentação (antixenose).

Lara, Sargo e Boiça Jr. (2000b) avaliaram a preferência alimentar de adultos de D iabrotica speciosa (Germar), pelos genótipos de batata Achat, Baronesa, Monalisa, N140-201 e NYL 235-4. Os resultados revelaram que o genótipo NYL 235-4 apresentou resistência do tipo não preferência para a alimentação em relação a D . speciosa.

Este trabalho teve como objetivo avaliar os efeitos de genótipos de batata sobre larvas e adultos de D. speciosa em condições de campo.

\section{MATERIAIS E MÉTODOS}

O experimento foi instalado no campo experimental da Cooperativa Castrolanda, em Castro-PR. Foram utilizadas cinco cultivares (Bintje, Jatte-Bintje, Monalisa, Atlantic e Crebella) e um clone (L 235-4) procedente da EMBRAPA - CNPH, desenvolvido na Universidade de Cornell, EUA.

Os tubérculos foram plantados em Latossolo Vermelho Amarelo, no ano de 2004, com espaçamento de 0,20 m e 0,75 m entre linhas com adubação de 3 t.ha $^{-1}$ da fórmula 4-16-8. As parcelas compunham-se de cinco linhas, com 5 metros de comprimento cada linha $\left(18,75 \mathrm{~m}^{2}\right)$. 
O delineamento experimental foi em blocos casualizados arranjados em parcelas subdivididas com doze tratamentos (parcela principal dois tratamentos: com e sem controle de insetos e sub-parcelas com seis tratamentos: cinco cultivares mais um clone) e quatro repetições para cada tratamento.

$\mathrm{Na}$ área tratada com inseticida foi aplicado, no sulco de plantio, 0 inseticida clorpirifós (2,880 L.ha-1) e o fungicida pencycuron (125 g.ha-1), com volume de calda de 400 L.ha $^{-1}$. Nas parcelas tratadas com inseticidas foram utilizados, em rotação semanal, os produtos: methamidophos 600CS, deltametrina 25CE, methomyl 215CS e carbaril 480SC.

O experimento foi avaliado comparando-se as duas áreas (tratada e não tratada com inseticidas), as diferenças entre as cultivares, e também as cultivares dentro das áreas tratadas e não tratadas, individualmente, com relação à desfolha da parte aérea e número de furos nos tubérculos ocasionados por adultos e larvas de D . speciosa.

As avaliações da desfolha foram feitas aos 29, 36 e 42 dias após a emergência das plantas e foram realizadas em 10 plantas.parcela ${ }^{-1}$ escolhidas aleatoriamente, sendo 3 folhas do estrato superior, 3 do estrato médio, 3 do estrato inferior e $1 \mathrm{com}$ estrato variável. Os danos foram quantificados pelas notas (em \% de área de área consumida): nota 0 , sem desfolha; nota 1, até 5\%; nota 2,5 - 10\%; nota 3, 10 - $15 \%$; nota 4, 15 - $20 \%$ e nota 5, 20 - 30\%. As análises estatísticas foram realizadas separadamente por data de avaliação. Para a avaliação dos danos das larvas de D . speciosa nos tubérculos foram contados os furos em 25 tubérculos por parcela.

O s dados obtidos durante 0 experimento foram submetidos à análise de variância conforme delineamento proposto e as médias foram comparadas pelo teste de TUKEY $(p<0,05)$.

\section{RESULTADOS}

Em todas as avaliações, considerando a planta inteira, ocorreram diferenças significativas entre os tratamentos na média geral de desfolha por D. speciosa, sendo a parcela com inseticida menos atacada que a parcela sem inseticida (Tabela 1), ou seja, os inseticidas utilizados diminuíram os danos causados pelos adultos da vaquinha.

TABELA 1 - Média geral de notas de desfolha ocasionada por adultos de D. speciosa, em três avaliações realizadas em plantas inteiras de cinco cultivares de batata e um clone, em Castro, PR

\begin{tabular}{llcc}
\hline & \multicolumn{3}{c}{ Dias após a emergência } \\
& $\mathbf{2 9}$ & $\mathbf{3 6}$ & $\mathbf{4 2}$ \\
\hline Plantas não tratadas com inseticidas & $1,52 \mathrm{a}$ & $2,12 \mathrm{a}$ & $2,02 \mathrm{a}$ \\
Plantas tratadas com inseticidas & $0,75 \mathrm{~b}$ & $0,96 \mathrm{~b}$ & $1,00 \mathrm{~b}$ \\
\hline CV (\%) & 41,21 & 26,07 & 24,50 \\
\hline
\end{tabular}

Médias seguidas por letras iguais não diferem entre si nas colunas (Tukey $\mathrm{p}>5 \%$ ).

Numberg et al. (1999) avaliaram o efeito da desfolha aos níveis de 25\%, 50\% e 75\% de área foliar das plantas. 0 nível de $25 \%$ de retirada das folhas, tanto aos 30 como aos 60 dias após a emergência, não resultou em redução da porcentagem de tubérculos. D esfolhas mais drásticas 50 e 75\% causaram redução de $20 \%$ no percentual de tubérculos graúdos. A batata é uma planta com alta capacidade de rebrota, o que permite que ela tolere altos níveis de desfolha durante o seu desenvolvimento, sem que haja reduções significativas na produção de tubérculos. 
De acordo com Cranshaw e Radcliffe (1980), a desfolha no estrato inferior é prejudicial no meio do ciclo da cultura; a desfolha no estrato superior ocasiona mais danos que no estrato intermediário e as maiores perdas na produção são verificadas quando a desfolha ocorre de maneira desuniforme, distribuída nos três estratos.

Cranshaw e Radcliffe (1980) constataram que plantas de batata possuem capacidade de regeneração quando a desfolha atinge até 33\% no período inicial da cultura e que, quando a desfolha ultrapassa $67 \%$ ocorre perda de produção.

Q uando se compara os dados de desfolha das vaquinhas adultas, entre as cultivares e o clone, considerando área tratada e não tratada em conjunto, constata-se que a variedade Crebella foi a mais atacada, embora esta diferença tenha sido significativa em relação às demais, apenas na primeira avaliação. 0 clone apresentou o menor nível de desfolha nas avaliações $2 \mathrm{e} 3 \mathrm{e}$ foi semelhante à maioria das cultivares na primeira avaliação (Tabela 2).

TABELA 2 - Média de notas atribuídas à desfolha provocada por adultos de D. speciosa em cinco cultivares de batata e um clone, em Castro, PR

\begin{tabular}{llll}
\hline & \multicolumn{3}{c}{ Dias após a emergência } \\
& $\mathbf{2 9}$ & $\mathbf{3 6}$ & $\mathbf{4 2}$ \\
\hline Crebella & $1,65 \mathrm{a}$ & $1,78 \mathrm{a}$ & $1,88 \mathrm{a}$ \\
Jatte Bintje & $1,25 \mathrm{~b}$ & $1,69 \mathrm{ab}$ & $1,40 \mathrm{~b}$ \\
Monalisa & $1,17 \mathrm{bc}$ & $1,66 \mathrm{ab}$ & $1,60 \mathrm{ab}$ \\
Atlantic & $0,99 \mathrm{bc}$ & $1,37 \mathrm{~b}$ & $1,61 \mathrm{ab}$ \\
Clone & $0,92 \mathrm{bc}$ & $1,01 \mathrm{c}$ & $1,05 \mathrm{c}$ \\
Bintje & $0,81 \mathrm{c}$ & $1,73 \mathrm{a}$ & $1,51 \mathrm{~b}$ \\
\hline F & 9,98 & 13,04 & 13,05 \\
CV (\%) & 41,21 & 26,07 & 24,50 \\
P & 0,00001 & 0,00001 & 0,00001 \\
\hline
\end{tabular}

Médias seguidas por letras iguais não diferem entre si nas colunas (Tukey $\mathrm{p}>5 \%$ ).

A variedade Bintje se comportou irregularmente, sendo a menos atacada na primeira avaliação e a mais atacada, junto com Crebella, na segunda avaliação. Esta diferença de desfolha entre as cultivares possivelmente ocorreu devido a uma questão de preferência do inseto em relação às cultivares testadas.

Salles (2000) identificou as cultivares que tiveram menor incidência, independente do tamanho do tubérculo, foram a Catucha, a Baraka e a A tlantic. As que tiveram maior incidência foram Aterix e Bintje. Nas cultivares de mesa a linhagem C-1485687 e cultivar Cristal foram as que tiveram maior quantidade de tubérculos enquadrados na categoria sem furo, diferindo das demais. A linhagem C-1485687 foi a menos atacada pelas larvas, sugerindo menor susceptibilidade à incidência de larvas de vaquinha entre as cultivares e linhagens de batata, tanto para a indústria como para a mesa.

Lara et al. (2004) avaliaram danos ocasionados por larvas e adultos de D . speciosa (G ermar) em tubérculos e folhas de 14 genótipos de batata. 0 s materiais observados apresentaram alta resistência média e materiais altamente suscetíveis como Achat, Bintje e o genótipo 288.719-13.

A análise dos danos nas folhas dentro da área não tratada e a análise comparativa entre a área tratada e não tratada com inseticidas, dentro de cada avaliação (Tabela 3), no que se refere aos danos na folhagem, revela que somente na primeira avaliação houve diferença significativa entre os tratamentos (Tabela 3). Q uando se observa a diferença entre as plantas tratadas com inseticidas aos 29 dias após a 
emergência vê-se que variedade Crebella foi a mais danificada e a variedade Bintje a menos atacada. Já na área tratada não se observou diferença entre os tratamentos, aos 29 dias após a emergência; observouse diferença significativa entre as áreas tratadas e não tratadas, com exceção da variedade Bintje, na qual não se observou diferenças entre as áreas citadas. Pode-se concluir que o uso de inseticidas só foi significativo na fase inicial da cultura. A pós este período, vários fatores podem ter contribuído para a não observância de efeito dos inseticidas: 0 aumento da massa foliar pode ter prejudicado a penetração da calda pulverizada nas plantas (ver observações sobre isso na tabela 3); houve redução na população dos adultos de vaquinha, assim como nas demais avaliações.

TABELA 3 - Comparação das médias de notas de desfolha (0 sem desfolha e 9 desfolha total) provocada por adultos de D . speciosa em diferentes cultivares de batata e um clone, entre plantas tratadas com inseticidas (T) e não tratadas com inseticidas (NT) em Castro, PR

\begin{tabular}{|c|c|c|c|c|c|c|}
\hline & \multicolumn{6}{|c|}{ Dias após a emergência } \\
\hline & \multicolumn{2}{|l|}{29} & \multicolumn{2}{|c|}{36} & \multicolumn{2}{|c|}{42} \\
\hline & NT & $\mathbf{T}$ & NT & $\mathbf{T}$ & NT & $\mathbf{T}$ \\
\hline Bintje & $0,97 \mathrm{a} \quad \mathrm{C}$ & 0,66 a A & 2,39 & 1,07 & 1,97 & 1,06 \\
\hline Jatte bintje & 1,68 а B & $0,83 \mathrm{~b} \mathrm{~A}$ & 2,40 & 0,98 & 2,02 & 0,78 \\
\hline Monalisa & 1,51 а $\mathrm{BC}$ & $0,83 \mathrm{~b} \mathrm{~A}$ & 2,11 & 1,22 & 1,99 & 1,21 \\
\hline Atlantic & 1,31 a $\mathrm{BC}$ & $0,66 \mathrm{~b} \mathrm{~A}$ & 1,92 & 0,83 & 2,20 & 1,01 \\
\hline Crebella & $2,30 \mathrm{aA}$ & $1,01 \mathrm{~b} \mathrm{~A}$ & 2,41 & 1,16 & 2,41 & 1,35 \\
\hline Clone & 1,34 a BC & $0,51 \mathrm{~b} \mathrm{~A}$ & 1,52 & 0,50 & 1,53 & 0,58 \\
\hline F & 2,0 & 80,0 & 1,0 & 50,0 & 1,0 & 35,0 \\
\hline CV \% & 41,0 & 21,0 & 26,0 & 70,0 & 24,0 & 50,0 \\
\hline $\mathrm{P}$ & 0.02 & 0,01 & 0,19 & 0,32 & 0.24 & 0,62 \\
\hline
\end{tabular}

Médias seguidas de letras minúsculas iguais não diferem entre si nas colunas, e seguidas por letras iguais maiúsculas, não diferem entre si na linha (Tukey $\mathrm{p}>5 \%$ ). Médias onde as letras não foram discriminadas, não diferem entre si.

Com relação aos danos nos tubérculos pôde-se constatar que a variedade Bintje foi a menos atacada, embora não tenha diferido de Jatte-Bintje, Crebella e o clone, e a variedade Monalisa a mais atacada (Tabela 4). Q uando se compara os danos nos tubérculos (Tabela 4) com a desfolha (Tabela 2), verifica-se, de modo geral, que a variedade Bintje e clone sofreram menos danos que outras cultivares. Já a variedade Crebella, embora tenha sido a mais danificada na folhagem, não foi a mais danificada nos tubérculos e a variedade Monalisa, medianamente danificada na folhagem, foi a mais danificada nos tubérculos. Estes dados demonstram que a preferência alimentar dos adultos da vaquinha nem sempre coincidem com a escolha do hospedeiro para a oviposição.

As cultivares oriundas da área tratada com inseticidas não apresentaram diferenças significativas entre si, e apresentaram danos menos significativos do que aqueles produzidos na área sem tratamento, apresentando diferenças estatísticas em relação à área sem tratamento (Tabela 4). 
TABELA 4 - Média de furos nos tubérculos proveniente de área tratada e não tratada com inseticidas, causado por larvas de D . speciosa em cinco cultivares de batata e um clone de batata, em Castro, PR

\begin{tabular}{lll}
\hline VARIEDADE & $\begin{array}{l}\text { Plantas não tratadas } \\
\text { com inseticidas }\end{array}$ & $\begin{array}{c}\text { Plantas tratadas } \\
\text { com inseticidas }\end{array}$ \\
\hline MONALISA & 58,66 a A & 7,28 a B \\
ATLANTIC & 47,91 b A & 2,60 a B \\
JATTE BINTJE & 41,98 bc A & 2,92 a B \\
CREBELLA & 39,20 bcd A & 7,73 a B \\
CLONE & 36,61 cd A & 3,47 a B \\
BINTJE & 31,07 d A & 4,85 a B \\
\hline F & 8,65 & 8,65 \\
CV $\%$ & 18,99 & 18,99 \\
P & 0,00007 & 0,00007 \\
\hline
\end{tabular}

Médias seguidas de letras minúsculas iguais não diferem entre si nas colunas, e seguidas por letras iguais maiúsculas, não diferem entre si na linha (Tukey $\mathrm{p}>5 \%$ ).

Com relação a esses danos (Tabela 4), dentro da área não tratada, com relação à comparação entre as cultivares, os resultados indicam uma possível diferença no nível de resistência entre as cultivares, sendo o clone e a Bintje as que obtiveram os melhores desempenhos, e a Monalisa e A tlantic as mais atacadas. Isto pode ter acontecido devido à uma preferência de oviposição do inseto por determinadas cultivares, em decorrência de diversos aspectos, como descritos por (LARA, 1991). Já dentro da área tratada não se encontrou diferença significativa entre as cultivares e o clone devido, provavelmente, à ação dos inseticidas.

\section{CONCLUSÕES}

Com base nos resultados e nas condições de realização do experimento pode-se concluir que:

1. a cultivar Crebella apresentou o maior índice de desfolha e a cultivar Bintje o menor;

2. com relação aos danos ocasionados pelos adultos, o uso de inseticidas só é justificado na fase inicial da cultura, sendo a variedade Crebella a mais suscetível e 0 clone o menos atacado;

3. com relação aos danos nos tubérculos a variedade Bintje foi a menos atacada e Monalisa a mais atacada;

4. o uso de inseticidas e reduziu significativamente os danos nos tubérculos, eliminando as diferenças entre as cultivares e 0 clone de batata;

5. não há relação entre os danos nas folhagens e os danos nos tubérculos.

\section{AGRADECIMENTOS}

Ao aluno Rogério O kuma, agradeço pela realização das atividades de coleta dos materiais a campo e auxílio operacional do projeto de pesquisa. 


\section{REFERÊNCIAS}

CRANSHAW, W. S.; RADCLIFFE, E. B. Effect of defoliation on yield of potatoes. J. Econ. Entomol., n. 73, p. 131-134, 1980.

G IBSO N, R. W.; TURNER, R. H. Insect trapping hairs on potato plants. Pest Artic. N ews Summ., v. 22, n. 2, p. 272-277, 1977.

HAJI, F. N. P. Biologia, danos e controle do adulto de Diabrotica speciosa (GE RMAR, 1824) (COLE OPTERA, CHRYSO MELIDAE), em cultura de batatinha (Solanum tuberosum L.). 1981. 53 f. Tese (D outorado em Entomologia) - Escola Superior de Agricultura Luiz de Q ueiroz, Universidade de São Paulo, Piracicaba, 1981.

LARA, F. M. Princípios de resistência de plantas a insetos. 2. ed. São Paulo: Ícone, 1991.

LARA, F. M.; POLETTI, M.; BARBO SA, J. C. Resistência de genótipos de batata (Solanum spp.) a D iabrotica speciosa (Germar, 1824) (Coleóptera: Chrysomelidae). Ciênc. Rural, Santa Maria, v. 30, n. 6, p. 927-931, 2000.

LARA, F. M.; SARG O, H. L. B.; BO IÇA JR, A. L. Preferência alimentar de adultos de Diabrotica speciosa (G ermar) (Coleóptera: Chrysomelidae) por genótipos de batata. An. Soc. Entomol. Brás., São Paulo, v. 29, n. 1, p. 131-137, 2000.

LARA, F. M. et al. Resistência de genótipos de batata e larvas e adultos de D iabrotica speciosa. Hortic. Brás., Brasília, v. 22, n. 4, p. 761-765, 2004.

NUMBERG, P. L. et al. Simulação de danos causados por insetos na planta de batata por meio de desfolhamento artificial. Ciênc. Agrotec., Lavras, v. 23, n. 2, p. 468-472, 1999.

OCHOA, C.; SCHMIEDICHE, P. Systemic exploitation and utilization of wild potato germplasm. In: INTERNATIONAL CONG RESS OF RESEARCH FOR THE POTATO IN THE YEAR, 1., 1983, 1983, Lima. Proceedings... Lima: W. Hooker, 1983. p. 142-144.

SALLES, L. A. Incidência de danos de D iabrotica speciosa em cultivares e linhagens de batata. Ciênc. Rural., Santa Maria, v. 30, n. 2, p. 205-209, 2000.

TINGEY, W. M.; G IBSO N, R.W. Feeding and mobility of the potato leafhopper impaired by glandular trichomes of Solanum berthaultii and S. polyadenium. J. Econ. Entomol., n. 71, p. 856-858, 1978.

TINGEY, W. M.; SINDEN, S. L. G landular puberscence, glycoalkaloid composition and resistance to the green peach aphid, potato leafhopper and potato fleabeetle in Solanum berthaultii. American Potato Journal, Oxford, v. 59, p. 95-106, 1982.

Recebido: 06/ 06/ 2008 Received: 06/ 06/ 2008

Aprovado: 05/ 12/ 2008

A pproved: 12/ 05/ 2008

Revisado: 19/ 08/ 2009

Reviewed: 08/ 19/ 2009 